\title{
Zur Kenntnis der «Schüttelinaktivierung» des Labs.
}

\section{Mitteilung.}

\author{
Von
}

Signe und Sigval Schmidt-Nielsen.

(Aus dem physiologischen Institut der Universität Christiania.)

(Der Redaktion zugegangen am 28. Mai 1909.)

Wie wir Ende des vorigen Jahres in einer vorläufigen Mitteilung haben zeigen können, ${ }^{1}$ ) gelingt es unter gewissen Umständen, das Labenzym durch einfaches Schütteln unwirksam zu machen. Über dies Phänomen, das, so weit wir wissen, eine bis da nie beschriebene Erscheinung darstellt, haben wir seit dem Frühjahr 1908 eine Reihe von Untersuchungen angestellt, worüber wir im folgenden berichten wollen.

Der Ausgangspunkt unserer Untersuchungen war die von uns zufällig gemachte auffällige Erscheinung, daß, wenn einige in einem Reagenzröhrchen eingeschlossenen Kubikzentimeter Lablösung (verdünnte Glycerinextrakte auf dem Labmagen des Kalbes) mit der Hand eine oder ein paar Minuten kräftig geschüttelt wurden, die milchkoagulierende Fähigkeit wenigstens um die Hälfte vermindert worden war, wie durch sofort angestellte Koagulationsversuche an Milch gezeigt werden konnte. Durch eine Reihe von hier nicht $\mathrm{zu}$ besprechenden Versuchen zeigte sich, daß diese "Schüttelinaktivierung», wie wir das Phänomen bezeichnet haben, nicht auf eine Alkaliwirkung des Glases (sie findet ebenfalls in einem Quarzrohre statt) oder auf Zufälligkeiten zurückzuführen war, sondern ein besonderes Phänomen darstellt, das immer auftritt, wenn neutrale Lablösungen aus Glycerinextrakten vom Labmagen des Kalbes be-

1) Signe og Sigval Schmidt-Niels en, Om mekanisk paavirkning af enzymer. Aarsberetning for det Biologiske Selskab i Kristiania for 1908, S. 45 u. f.; Nyt Mag. f. Naturv. Bd. XLVII. 
reitet, kräftig geschüttelt werden, und, so weit wir jetzt beobachtet haben, auch mit anderen Lablösungen sich zeigen läßt, wenn verschiedene Versuchsbedingungen genügend berücksichtigt werden.

Auf den folgenden Seiten werden wir hauptsächlich Versuche besprechen, die die Abhängigkeit der Schüttelinaktivierung von der Dauer und der Geschwindigkeit des Schüttelns, der Enzymkonzentration, der Versuchstemperatur und von ähnlichen Versuchsbedingungen zeigen. Hierzu kamen nur von uns selbst aus Kalbsmagen mittels Glycerinextraktion dargestellte Lablösungen zur Verwendung. Das Verhalten anderer Lablösungen (z. B. der Präparate des Handels), und die Beeinflussung der Schüttelinaktivierung durch verschiedene in kleinen Mengen anwesende Säuren und chemisch indifferente Stoffe, sowie die unter gewissen Versuchsbedingungen eintretende Reversibilität des Prozesses wird in folgenden Mitteilungen Erwähnung finden.

\section{Versuchsanordnung.}

Die Glycerinextrakte wurden in der Weise bereitet, daß frische Labmagen von jungen Kälbern zuerst mit Wasser genau abgespült wurden, und danach von den Falten des Fundusteiles die Drüsenschicht (nebst dem Schleime) mit einem Urglase abgeschabt und in der zehnfachen Menge wasserhaltigen neutralen Glycerins verteilt und vor dem Filtrieren ein paar Tage hingestellt wurden. Es zeigte sich in den meisten Fällen, daß diese Extrakte fast ausschließlich Chymosin enthielten, und sie waren deswegen ohne Aktivierung mittels Säuren für die Schüttelversuche verwendbar. Sie wurden zuerst so weit verdünnt, daß sie in dem Verhältnis $2 \mathrm{ccm}$ Enzymlösung auf $10 \mathrm{ccm}$ Milch bei $37^{\circ}$ Gerinnung derselben in ca. 8 Minuten hervorrief; hierzu war für die verschiedenen Extrakte eine Verdünnung mit Wasser von 2-4 ccm Glycerinextrakt auf $100 \mathrm{ccm}$ notwendig. Als Maß für die in den verschiedenen Proben anwesende Labmenge ist stets die Koagulationsfähigkeit gegen völlig frische Kuhmilch $(2: 10)$ verwendet worden. Es war nicht zu vermeiden, daß die Eigenschaften der Milch an verschiedenen Tagen variierten, was aus den Anfangswerten der 
mit Lablösungen derselben Konzentration ausgeführten Versuche ersichtlich ist. Diese Variationen sind jedoch ohne praktische Bedeutung, wenn für die Beurteilung der Versuche die zurückgebliebene Enzymmenge mit Hilfe des Zeitgesetzes des Labs berechnet wird. Es muß hervorgehoben werden, daß die Bestimmung der zurückgebliebenen Labmenge stets sofort an den direkt nach dem beendeten Schütteln herausgenommenen Proben ausgeführt wurde, und daß die angeführten Koagulationszeiten den Mittelwert von mehreren sofort nach einander angestellten Koagulationsversuchen darstellen.

In den ersten orientierenden Versuchen geschah die mechanische Bewegung der Lablösungen in der Weise, daß sie in einem Reagenzröhrchen eingeschlossen ganz einfach mit der Hand kräftig geschüttelt wurden. Für ein quantitatives Studium des Phänomens war dies natürlich, was sich auch experimentell herausstellte, ein zu unsicheres und wenig gleichmäßiges Verfahren, weshalb sie durch rein maschinelle Anordnungen ersetzt wurde. Nach verschiedenen Versuchen blieben wir für die in dieser Mitteilung zu erwähnenden Versuche bei der folgenden Anordnung stehen:

In der feststehenden aus bestem Jenaer Verbrennungsglas verfertigten Röhre wurde ein Rührer, aus vier durchlöcherten Ebonitplatten an einer silbernen Stange befestigt, schnell auf und ab bewegt. Die Zahl der Bewegungen konnte nach Belieben zwischen einigen wenigen und etwa 250 pro Minute variiert werden. In dem ca. $40 \mathrm{ccm}$ fassenden Röhrchen befanden sich $25 \mathrm{ccm}$ Flüssigkeit, die durch die Bewegungen des Rührers heftig mit Luft geschüttelt wurden.

Damit die Versuche bei einer gleichmäßigen Temperatur stattfinden sollten, war das Versuchsröhrchen in ein Wasserbad eingesenkt. Wo nichts anderes angeführt ist, betrug die Versuchstemperatur $16^{\circ} \mathrm{C}$.

Vor jedem Versuche wurde das Versuchsröhrchen mit destilliertem Wasser ausgekocht, und der Rührer mit kochend heißem Wasser sorgfältig abgespült. Wir haben uns überhaupt bemüht, die einzelnen Versuche so gleichförmig wie möglich anzustellen. 
Der Einfluß der Dauer des Schüttelns.

Durch unsere ersten Versuche stellte sich heraus, daß die Dauer des Schüttelns auf die Größe der «Inaktivierung» einen bedeutenden Einfluß ausübt, und zwar in der Weise, daß, je länger die Schüttelzeit dauert, um so stärker auch die «Inaktivierung» ist.

Tabelle I.

Einfluß der Schütteldauer.

\begin{tabular}{c|c|c|c|c|c}
\hline \hline $\begin{array}{c}\text { Dauer des } \\
\text { Schüttelns } \\
\text { Minuten }\end{array}$ & $\begin{array}{c}\text { Koagulations- } \\
\text { zeit } \\
\text { Minuten }\end{array}$ & $(\mathrm{a}-\mathrm{x})$ & $\mathrm{k}_{\mathbf{1}}$ & $\mathrm{k}_{\mathbf{2}}$ & $\mathrm{k}_{\frac{3}{2}}$ \\
\hline 0 & 8,5 & - & - & - & - \\
1,0 & 24,0 & 35,4 & 0,45 & 1,8 & 1,4 \\
2,0 & 46,0 & 18,5 & 0,37 & 2,2 & 1,3 \\
2,5 & 57,0 & 14,9 & 0,33 & 2,3 & 1,3 \\
3,0 & 68,5 & 12,4 & 0,30 & 2,4 & 1,2 \\
3,5 & 84 & 10,1 & 0,28 & 2,5 & 1,2 \\
4,0 & 101 & 8,4 & 0,27 & 2,7 & 1,2 \\
5,0 & 176 & 4,8 & 0,26 & 3,9 & 1,4
\end{tabular}

Tabelle II.

Einfluß der Schütteldauer.

\begin{tabular}{c|c|c|c|c|c}
\hline $\begin{array}{c}\text { Dauer des } \\
\text { Schüttelns } \\
\text { Minuten }\end{array}$ & $\begin{array}{c}\text { Koagulations- } \\
\text { zeit } \\
\text { Minuten }\end{array}$ & $(\mathrm{a}-\mathrm{x})$ & $\mathbf{k}_{\mathbf{1}}$ & $\mathbf{k}_{\mathbf{2}}$ & $\frac{\mathbf{k}_{\mathbf{3}}}{\mathbf{2}}$ \\
\hline 0 & 9,5 & - & - & - & - \\
1,0 & 21,5 & 44,2 & 0,35 & 1,3 & 1,01 \\
1,5 & 29,0 & 32,8 & 0,32 & 1,4 & 1,00 \\
2,0 & 33,7 & 28,2 & 0,27 & 1,3 & 0,88 \\
3,0 & 51 & 18,6 & 0,24 & 1,5 & 0,88 \\
4,0 & 74 & 12,8 & 0,22 & 1,7 & 0,90 \\
5,0 & 110 & 8,6 & 0,21 & 2,1 & 0,96 \\
6,0 & 155 & 6,1 & 0,20 & 2,6 & 1,01
\end{tabular}


In den Tabellen I und II haben wir zwei einschlägige Versuchsserien wiedergegeben. Wenn die ursprünglich anwesende Labmenge mit $a$ bezeichnet wird, und $x$ die Labmenge bedeutet, die nach einer gewissen Zeit durch die Reaktion in Anspruch genommen ist, wird durch $(a-x)$ die zurückgebliebene Labmenge ausgedrückt. Sie ist in unseren Tabellen in Prozenten der ursprünglichen Labmenge angegeben.

Man ersieht, daß nach dem Schütteln von nur 5 oder 6 Minuten Dauer fast alles Lab, und daß binnen der ersten Minute mehr als die Hälfte des Labs «inaktiviert» worden ist.

Sowohl bei graphischer Aufzeichnung dieser Versuche, wie überhaupt der experimentell gefundenen Daten, erhält man stets regelmäßig verlaufende Kurven von ähnlicher Form, wie wir sie früher $^{1}$ ) für die monomolekuläre Destruktion des Labs durch Licht gefunden haben. Die stets gefundene regelmäßige Form der verschiedenen Kurven deutet darauf hin, daß die Reaktion nach bestimmten Gesetzen verläuft.

Um in diese einen Einblick zu bekommen, haben wir die Reaktionskonstante in der Formel $\frac{d x}{d t}=k(a-x)^{n}$, und zwar nicht nur mit $\mathrm{n}$ gleich 1 , sondern auch mit $\mathrm{n}$ gleich 2 und $3 / 2$ ausgerechnet. Wenn $t_{0}$ die Koagulationszeit der nicht behandelten Lablösung ist und $t_{1}$ die der in der Zeit $\tau$ geschüttelte Probe angibt, und wenn vorausgesetzt wird, daß stets $a \cdot t_{0}=(a-x) \cdot t_{1}$ ist, erhält man für die Berechnung der Reaktionskonstanten die folgenden Ausdrücke:

Bei $n=1$ : die Reaktionskonstante $=\frac{1}{\tau} \cdot \log \frac{t_{1}}{t_{0}}=k_{1}$

$$
\begin{aligned}
& \text { " } \mathrm{n}=2 \text { : 》 } \\
& =\frac{1}{\tau}\left(\frac{t_{1}}{t_{0}}-1\right)=k_{2} \\
& \text { 》 } \mathrm{n}=\frac{3}{2}: \\
& =\frac{2}{\tau}\left(\sqrt{\frac{t_{1}}{t_{o}}}-1\right)=\frac{k_{3}}{2}
\end{aligned}
$$

indem bei den zwei letzten Konstanten der Wert von a gleich 1

1) Signe und Sigval Schmidt-Nielsen, Quantitative Versuche über die Destruktion des Labs durch Licht. III. Mitteilung. Diese Zeitschrift, Bd. LVIII, S. $233 \mathrm{ff}$. 
angenommen wird. Bei der Rechnung sind dekadischeLogarithmen verwendet worden.

Aus den in den Tabellen I-III angeführten Werten für die Reaktionskonstanten ist ersichtlich, daß es sich jedenfalls nicht um eine bimolekuläre Reaktion handeln kann. Der in der Tabelle III wiedergegebene Versuch stimmt in ihrer Abhängigkeit der Schüttelzeit völlig mit dem Verlaufe einer monomolekulären Reaktion überein.

Tabelle III.

Einfluß der Schütteldauer.

\begin{tabular}{c|c|c|c|c|c}
\hline $\begin{array}{c}\text { Dauer des } \\
\text { Schüttelns } \\
\text { Minuten }\end{array}$ & $\begin{array}{c}\text { Koagulations- } \\
\text { zeit } \\
\text { Minuten }\end{array}$ & $(\mathrm{a}-\mathrm{x})$ & $\mathrm{k}_{\mathbf{1}}$ & $\mathrm{k}_{\mathbf{2}}$ & $\mathbf{k}_{\mathbf{3}}$ \\
\hline 0 & 8,15 & - & - & - & - \\
2,0 & 20,0 & 40,8 & 0,19 & 0,73 & 0,28 \\
3,0 & 24,3 & 33,5 & 0,16 & 0,66 & 0,24 \\
4,0 & 38,0 & 21,4 & 0,17 & 0,92 & 0,29 \\
5,0 & 67,5 & 12,1 & 0,18 & 1,4 & 0,38 \\
6,0 & 104 & 7,8 & 0,18 & 2,0 & 0,43
\end{tabular}

Indessen ist aus den Tabellen I und II sowie aus den meisten in dieser Abhandlung wiedergegebenen Tabellen ersichtlich, daß der Verlauf der Reaktion in ihrer Abhängigkeit von der Zeit komplizierter ist, als der monomolekulären Reaktionsformel entsprechen würde. Die experimentellen Daten stimmen in den meisten Fällen am besten mit der Reaktionsformel $\frac{d x}{d t}=k(a-x)^{\frac{3}{2}}$ überein; sie hat jedoch, wie aus den in den folgenden Abschnitten angeführten Versuchsreihen hervorgeht, die Neigung, bei geringerer Enzymkonzentration und erhöhter Temperatur monomolekulär zu werden. In einem einzigen Falle (und dies bei hoher Konzentration) stimmte der Reaktionsverlauf mit der bimolekulären Formel.

Auf diesen Befund kann selbstverständlich vorläufig kein besonderes Gewicht gelegt werden. Die das Phänomen be- 
dingenden Faktoren (z. B. Einfluß der Verunreinigungen) sind nämlich bis jetzt viel zu wenig erforscht, und die Möglichkeit kann nicht ausgeschlossen werden, daß es sich nicht um ein einziges, sondern um mehrere neben einander verlaufende Prozesse handelt. Wenn dem so wäre, wird natürlich, je nach dem Vorherrschen des einen oder anderen Prozesses, der Gesamtprozeß zeitlich verschieden verlaufen können.

Ein Zufall kann es jedoch auf der anderen Seite kaum sein, daß wir durchgehend die Reaktionsformel $\frac{d x}{d t}=k(a-x)^{\frac{3}{2}}$ verwendbar finden. Dies ist selbstverständlich nur ein Ausdruck für die experimentellen Beobachtungen, und es darf hiermit irgend eine Vorstellung über die Natur des Vorganges nicht verbunden werden.

Die einzige Schlußfolgerung, die wir zurzeit hieraus ziehen, ist die, daß wir mit der durch diese Berechnung gefundenen Reaktionskonstante einen Vergleich mit dem Einfluß anderer Versuchsmomente, z. B. Enzymkonzentration, Temperatur und Schüttelgeschwindigkeit anstellen können. Und dies ist ein großer Vorteil, denn die Werte $(a-x)$ können bei verschiedenen Schüttelzeiten nicht verglichen werden, und sind auch nicht hierzu so geeignet wie die Reaktionskonstante.

Einfluß der Geschwindigkeit des Schüttelns.

Die Versuche über den Einfluß der Schüttelgeschwindigkeit wurden in der Weise angestellt, daß bei einer konstanten Schüttelzeit von 2 Minuten die Zahl der Schüttelungen pro Minute variiert wurde. Die Größe der Bewegungen des Rührers und die sonstige Versuchsanordnung war hierbei völlig unverändert.

Zwei solche Versuchsreihen, die mit verschiedenen Lablösungen angestellt wurden, sind in den Tabellen IV und V wiedergegeben. Damit die Unterschiede besser hervortreten sollen, sind sowohl die Werte $(a-x)$, wie die beiden Reaktionskonstanten $\mathrm{k}_{1}$ und $\mathrm{k}_{\frac{3}{2}}$, angeführt. 
Zur Kenntnis der «Schüttelinaktivierung» des Labs. I.

Tabelle IV.

Einfluß der Schüttelgeschwindigkeit.

\begin{tabular}{c|c|c|c|c}
\hline $\begin{array}{c}\text { Zahl der } \\
\text { Schüttelungen } \\
\text { pro Minute }\end{array}$ & $\begin{array}{c}\text { Koagulations- } \\
\text { zeit } \\
\text { Minuten }\end{array}$ & $(\mathrm{a}-\mathrm{x})$ & $\mathbf{k}_{\mathbf{1}}$ & $\mathbf{k}_{\mathbf{3}}$ \\
\hline 0 & 7,8 & - & - & - \\
116 & 9,0 & 86,7 & 0,03 & 0,04 \\
155 & 16,8 & 46,4 & 0,17 & 0,23 \\
190 & 25,5 & 30,6 & 0,26 & 0,40 \\
240 & 39,5 & 19,7 & 0,35 & 0,63 \\
300 & 41,5 & 18,8 & 0,36 & 0,65
\end{tabular}

Tabelle V.

Einfluß der Schüttelgeschwindigkeit.

\begin{tabular}{c|c|c|c|c}
\hline $\begin{array}{c}\text { Zahl der } \\
\text { Schüttelungen } \\
\text { pro Minute }\end{array}$ & $\begin{array}{c}\text { Koagulations- } \\
\text { zeit } \\
\text { Minuten }\end{array}$ & $(\mathrm{a}-\mathrm{x})$ & $\mathrm{k}_{\mathrm{t}}$ & $\frac{\mathrm{k}_{\mathbf{8}}}{\mathrm{z}}$ \\
\hline 0 & 6,9 & - & - & - \\
100 & 9,9 & 69,7 & 0,08 & 0,10 \\
118 & 11,7 & 59,0 & 0,12 & 0,15 \\
131 & 13,0 & 53,1 & 0,14 & 0,19 \\
146 & 25,6 & 27,0 & 0,29 & 0,46 \\
165 & 45,0 & 15,3 & 0,41 & 0,78 \\
183 & 53,5 & 12,9 & 0,45 & 0,89 \\
203 & 59,0 & 11,7 & 0,47 & 0,96 \\
209 & 61,5 & 11,2 & 0,48 & 0,99 \\
219 & 61,5 & 11,2 & 0,48 & 0,99 \\
231 & 63,0 & 11,0 & 0,48 & 1,01
\end{tabular}

Man sieht, daß die Wirkung im Anfange mit der vermehrten Geschwindigkeit rasch zunimmt, um bald einen asymptotischen Wert anzunehmen.

Nach dieser Erfahrung sind unsere späteren Versuche mit einer Zahl der minutlichen Schüttelungen von gegen 250 an- 
gestellt worden, indem bei dieser Höhe derselben der Einfluß kleiner, trotz aller Sorgfalt nicht zu vermeidenden Variationen vernachlässigt werden kann.

Es wäre gewiß von großem Interesse, eine nähere Beziehung zwischen der mechanischen Arbeit und dem numerischen Werte der «Inaktivierung» kennen zu lernen, aber wir haben bis zur weiteren Aufklärung der generellen Seiten des Phänomens darauf verzichtet.

Einfluß der Enzymkonzentration.

Wenn bei ein und derselben Schüttelgeschwindigkeit verschiedene Lablösungen gleich lange geschüttelt werden, zeigt sich, daß die Lösungen in verschiedenem Maße inaktiviert werden, und zwar in der Weise, daß die Wirkung mit der verminderten Enzymkonzentration zunimmt.

Tabelle VI.

Einfluß der Enzymkonzentration.

\begin{tabular}{c|c|c|c}
\hline $\begin{array}{c}\text { Enzym- } \\
\text { konzentration }\end{array}$ & $(\mathrm{a}-\mathrm{x})$ & $\mathrm{k}_{\mathrm{i}}$ & $\mathrm{k}_{\frac{\mathbf{s}}{\mathbf{2}}}$ \\
\hline 2 & 14,8 & 0,41 & 0,80 \\
5 & 20,9 & 0,34 & 0,59 \\
12,5 & 60,0 & 0,11 & 0,15 \\
25 & 85 & 0,03 & 0,04
\end{tabular}

In der Tabelle VI ist ein solcher Konzentrationsversuch wiedergegeben. Er wurde in der Weise ausgeführt, daß von ein und demselben Labextrakte je $2 \mathrm{ccm}, 5 \mathrm{ccm}, 12,5 \mathrm{ccm}$ und $25 \mathrm{ccm}$ mit Wasser auf $250 \mathrm{ccm}$ verdünnt wurden. Diese Lösungen, die also in arbiträren Einheiten Konzentrationen von $2-5-12,5$ und 25 repräsentieren, wurden 2 Minuten lang in genau derselben Weise geschüttelt und die Koagulationszeiten sofort bestimmt. In der Tabelle sind nur die hieraus berechneten Werte von $(a-x)$, sowie die Reaktionskonstante $k_{1}$ und $\mathrm{k}_{\frac{3}{2}}$ angeführt worden. Man bekommt hieraus einen guten Eindruck von dem Einfluß der Konzentration.

Da der Einfluß der Schüttelzeit nicht sicher festgestellt ist, konnten wir uns natürlich nicht mit Beobachtungen bei 
einer einzelnen Schüttelzeit begnügen, sondern mußten bei verschiedenen Enzymkonzentrationen Versuche mit variierenden Schüttelzeiten anstellen.

In den folgenden Tabellen VII-X haben wir solche vollständige Serien bei den arbiträr gewählten Enzymkonzentrationen 10-7,5-5 und 2,5 ausgeführt zusammengestellt. Sie beziehen sich auf dasselbe Labextrakt, wovon je $10 \mathrm{ccm}, 7,5 \mathrm{ccm}, 5 \mathrm{ccm}$ und $2,5 \mathrm{ccm}$ mit Wasser auf $250 \mathrm{ccm}$ verdünnt wurden. Diese Lösungen sind dann in den für jede Versuchsserie angeführten Zeiten mit einer Geschwindigkeit des Rührers von ca. $245 \mathrm{Be}-$ wegungen pro Minute geschüttelt worden. Die in diesen Versuchen erhaltenen Werte sind nicht direkt mit denen der Tabelle VI vergleichbar, indem sie sich auf verschiedene Labextrakte beziehen.

Tabelle VII.

Versuch bei der Enzymkonzentration 10.

\begin{tabular}{c|c|c|c|c}
\hline \hline $\begin{array}{c}\text { Schüttelzeit } \\
\text { Minuten }\end{array}$ & $\begin{array}{c}\text { Koagulations- } \\
\text { zeit } \\
\text { Minuten }\end{array}$ & $(\mathrm{a}-\mathrm{x})$ & $\mathbf{k}_{\mathbf{1}}$ & $\frac{\mathbf{k}_{\mathbf{8}}}{\mathbf{8}}$ \\
\hline 0 & 5,0 & - & - & - \\
3,0 & 8,5 & 58,8 & 0,077 & 0,101 \\
4,0 & 9,15 & 54,6 & 0,066 & 0,088 \\
6,0 & 10,75 & 46,5 & 0,055 & 0,078 \\
8,0 & 12,85 & 38,9 & 0,051 & 0,075 \\
10,0 & 15,9 & 31,4 & 0,050 & 0,078 \\
15,0 & 19,5 & 25,6 & 0,039 & 0,065
\end{tabular}

Tabelle VIII.

Versuch bei der Enzymkonzentration 7,5.

\begin{tabular}{c|c|c|c|c}
\hline \hline $\begin{array}{c}\text { Schüttelzeit } \\
\text { Minuten }\end{array}$ & $\begin{array}{c}\text { Koagulations- } \\
\text { zeit } \\
\text { Minuten }\end{array}$ & $(\mathrm{a}-\mathrm{x})$ & $\mathbf{k}_{\mathbf{1}}$ & $\mathbf{k}_{\frac{\mathbf{3}}{\mathbf{2}}}$ \\
\hline 0 & 6,25 & - & - & - \\
2,0 & 14,35 & 43,6 & 0,18 & 0,26 \\
4,0 & 23,55 & 26,5 & 0,14 & 0,24 \\
5,0 & 24,4 & 25,6 & 0,12 & 0,20
\end{tabular}


Tabelle IX.

Versuch bei der Enzymkonzentration 5.

\begin{tabular}{c|c|c|c|c}
\hline \hline Schüttelzeit & $\begin{array}{c}\text { Koagulations- } \\
\text { zeit } \\
\text { Minuten }\end{array}$ & $(\mathrm{a}-\mathrm{x})$ & $\mathrm{k}_{\mathbf{1}}$ & $\mathrm{k}_{\mathbf{3}}$ \\
\hline 0 & 6,13 & - & - & - \\
1,0 & 11,85 & 51,7 & 0,29 & 0,39 \\
2,0 & 22,55 & 27,2 & 0,28 & 0,46 \\
3,0 & 29,9 & 20,5 & 0,23 & 0,40 \\
5,0 & 42,85 & 14,3 & 0,21 & 0,41
\end{tabular}

Tabelle X.

Versuche bei der Enzymkonzentration 2,5.

\begin{tabular}{c|c|c|c|c}
\hline \hline Schüttelzeit & $\begin{array}{c}\text { Koagulations- } \\
\text { zeit } \\
\text { Minuten }\end{array}$ & $(\mathrm{a}-\mathbf{x})$ & $\mathbf{k}_{\mathbf{1}}$ & $\mathbf{k}_{\frac{\mathbf{3}}{\mathbf{z}}}$ \\
\hline 0 & 12,73 & - & - & - \\
1,0 & 31,65 & 40,2 & 0,40 & 0,58 \\
2,0 & 68,45 & 18,6 & 0,37 & 0,66 \\
2,5 & 78,2 & 16,3 & 0,32 & 0,59 \\
3,0 & 106,5 & 12,0 & 0,31 & 0,63
\end{tabular}

Aus den aufgeführten Daten geht hervor, sowohl wenn man die Werte $(a-x)$ als die Reaktionskonstante vergleicht, daß die Wirkung bei den verdünnteren Lösungen eine größere ist als bei den mehr konzentrierten. Bei graphischer Aufzeichnung stellen die gefundenen Werte regelmäßige Kurven dar.

Man wird gegen diese Versuche einwenden können, daß nicht nur die Labmenge, sondern auch der Gehalt der Verunreinigungen und besonders der des Glycerins in den obenstehenden Versuchsreihen verschieden war und die Resultate vielleicht nur einen Ausdruck für den verminderten Glyceringehalt darstellen. Um diesem Einwand entgegenzutreten, wurde eine zweite Reihe von Versuchen (die Tabellen XI-XIII) angestellt, wo durch Hinzufügung von Glycerin (resp. $2,5 \mathrm{ccm}$, 
Zur Kenntnis der «Schüttelinaktivierung» des Labs. I.

$5 \mathrm{ccm}$ und 7,5 ccm) für die nämliche Glycerinkonzentration (etwa 10:250) in den verdünnten Lösungen gesorgt worden war.

Tabelle XI.

Versuche bei der Enzymkonzentration 7,5 (mit Glycerinzusatz).

\begin{tabular}{c|c|c|c|c}
\hline \hline $\begin{array}{c}\text { Schüttelzeit } \\
\text { Minuten }\end{array}$ & $\begin{array}{c}\text { Koagulationszeit } \\
\text { Minuten }\end{array}$ & $(\mathrm{a}-\mathrm{x})$ & $\mathbf{k}_{\mathbf{1}}$ & $\mathbf{k}_{\frac{\mathbf{3}}{\mathbf{2}}}$ \\
\hline 0,0 & 6,75 & - & - & - \\
2,0 & 11,85 & 57,0 & 0,12 & 0,16 \\
3,0 & 16,2 & 41,7 & 0,13 & 0,18 \\
4,0 & 19,9 & 33,9 & 0,12 & 0,18 \\
6,0 & 27,9 & 24,2 & 0,10 & 0,17 \\
8,0 & 32,85 & 20,6 & 0,09 & 0,15
\end{tabular}

Tabelle XII.

Versuch bei der Enzymkonzentration 5 (mit Glycerinzusatz).

\begin{tabular}{c|c|c|c|c}
\hline $\begin{array}{c}\text { Schüttelzeit } \\
\text { Minuten }\end{array}$ & $\begin{array}{c}\text { Koagulationszeit } \\
\text { Minuten }\end{array}$ & $(\mathrm{a}-\mathrm{x})$ & $\mathrm{k}_{\mathbf{1}}$ & $\mathrm{k}_{\mathbf{3}}$ \\
\hline 0,0 & 6,43 & - & - & - \\
2,0 & 12,75 & 50,4 & 0,15 & 0,20 \\
3,0 & 17,4 & 37,0 & 0,14 & 0,22 \\
4,0 & 22,9 & 28,1 & 0,14 & 0,22 \\
5,0 & 26,6 & 24,2 & 0,12 & 0,21
\end{tabular}

Tabelle XIII.

Versuch bei der Enzymkonzentration 2,5 (mit Glycerinzusatz).

\begin{tabular}{c|c|c|c|c}
\hline $\begin{array}{c}\text { Schüttelzeit } \\
\text { Minuten }\end{array}$ & $\begin{array}{c}\text { Koagulationszeit } \\
\text { Minuten }\end{array}$ & $(\mathrm{a}-\mathrm{x})$ & $\mathrm{k}_{\mathbf{1}}$ & $\frac{\mathbf{k}_{\mathbf{3}}}{\mathbf{2}}$ \\
\hline \multirow{2}{*}{0} & 13,7 & - & - & - \\
1,0 & 22,4 & 61,2 & 0,21 & 0,28 \\
2,0 & 34,15 & 40,1 & 0,20 & 0,29 \\
3,0 & 45,25 & 30,3 & 0,17 & 0,27 \\
4,0 & 70,0 & 19,6 & 0,18 & 0,32
\end{tabular}


In Übereinstimmung mit dem oben Gefundenen geht aus diesen Versuchen hervor, daß die Wirkung um so größer ist, je geringer die Enzymkonzentration. Außerdem ist aus diesen Versuchen ersichtlich, daß das Glycerin in den verwendeten Konzentrationen auf die "Inaktivierung» hindernd gewirkt hat.

Um dies Verhalten besser hervortreten zu lassen, haben wir für die in den Tabellen VII bis XIII angeführten Versuchsreihen die mittleren Reaktionskonstanten $\mathrm{k}_{\frac{3}{2}}$ berechnet und in der Tabelle XIV zusammengestellt, indem es hierdurch ermöglicht wird, einen direkten Vergleich anzustellen, durch welchen man einen ungefähren Begriff von den numerischen Werten des Einflusses der Enzymkonzentration resp. des Glycerins erhält. Es muß jedoch daran erinnert werden, daß diese Werte nur approximative sind.

Tabelle XIV.

Einfluß der Enzymkonzentration.

\begin{tabular}{|c|c|c|}
\hline $\begin{array}{c}\text { Enzym- } \\
\text { konzentration }\end{array}$ & $\begin{array}{l}\text { Versuche } \\
\text { mit konstantem } \\
\text { Glyceringehalt (10) } \\
\text { Reaktionskonstante }\end{array}$ & $\begin{array}{c}\text { Versuche } \\
\text { mit fallendem Glycerin- } \\
\text { gehalt }(10-2,5) \\
\text { Reaktionskonstante }\end{array}$ \\
\hline 10,0 & etwa 0,08 & etwa 0,08 \\
\hline 7,5 & $» \quad 0,17$ & » 0,23 \\
\hline 5,0 & \ 0,21 & $\diamond \quad 0,41$ \\
\hline 2,5 & $\gg \quad 0,29$ & $\gtrsim \quad 0,61$ \\
\hline
\end{tabular}

Einfluß der Reaktionstemperatur.

Um den Einfluß der Versuchstemperatur näher studieren zu können, konnten natürlich nur diejenigen Temperaturen zur Verwendung kommen, welche an und für sich ohne schädigende Einwirkung auf das Lab sind. Nach den Untersuchungen von Madsen und Walbum ${ }^{1}$ ) dürfte eine Temperaturschädi-

1) Th. Madsen et L. Walbum, Recherches sur l'affaiblissement de la présure. Festschrift für Ol of Hammarsten, 1906, Nr. X, Uppsala, Läkareför. Förh., Bd. XI, Suppl. 
gun erst oberhalb $37^{\circ}$ eintreten. Wir haben jedoch nur Temperturen bis zu $30^{\circ} \mathrm{C}$. verwendet. Bezüglich der Einzelheiten dieer Versuche brauchen wir wohl kaum anzuführen, daß die Schttelung erst dann stattfand, nachdem das Versuchsröhrche mit der Lablösung die Temperatur des Wasserbades angenmmen hatte.

Bei den Temperaturen $0,10,20$ und $30^{\circ} \mathrm{C}$. wurden vollstädige Serien mit verschiedener Schüttelzeit, aber mit dersellen Schüttelgeschwindigkeit und derselben Labkonzentratior.(5 : 250 desselben Labextraktes) angestellt. Die gewonnenen Datn sind in den Tabellen XV-XVIII zusammengestellt.

Tabelle XV.

Versuche bei einer Versuchstemperatur von $\mathbf{0}^{\circ} \mathrm{C}$.

\begin{tabular}{c|c|c|c|c}
\hline $\begin{array}{c}\text { Schittelzeit } \\
\text { Mnuten }\end{array}$ & $\begin{array}{c}\text { Koagulationszeit } \\
\text { Minuten }\end{array}$ & $(\mathrm{a}-\mathrm{x})$ & $\mathrm{k}_{\mathrm{i}}$ & $\mathrm{k}_{\frac{\mathbf{3}}{\mathrm{z}}}$ \\
\hline 0 & 9,2 & - & - & - \\
1,0 & 14,4 & 63,9 & 0,19 & 0,25 \\
2,0 & 20,2 & 45,5 & 0,17 & 0,24 \\
3,0 & 28,2 & 32,6 & 0,16 & 0,25 \\
4,0 & 35,0 & 26,3 & 0,15 & 0,24 \\
5,0 & 41,4 & 22,2 & 0,13 & 0,22 \\
6,0 & 52,5 & 17,5 & 0,13 & 0,23
\end{tabular}

Tabelle XVI.

Versuche bei einer Versuchstemperatur von $10^{\circ} \mathrm{C}$.

\begin{tabular}{c|c|c|c|c}
\hline $\begin{array}{c}\text { Schittelzeit } \\
\text { Mnuten }\end{array}$ & $\begin{array}{c}\text { Koagulationszeit } \\
\text { Minuten }\end{array}$ & $(\mathrm{a}-\mathrm{x})$ & $\mathbf{k}_{\mathbf{1}}$ & $\frac{\mathbf{k}_{\mathbf{3}}}{\mathbf{z}}$ \\
\hline & 7,9 & - & - & - \\
0,0 & 23,5 & 33,6 & 0,24 & 0,36 \\
2,0 & 30,35 & 26,0 & 0,20 & 0,32 \\
3,0 & 46,25 & 17,1 & 0,19 & 0,36 \\
4,0 & 56,85 & 13,9 & 0,17 & 0,36 \\
5,0 & 95,5 & 8,3 & 0,18 & 0,41 \\
6,0 & & & &
\end{tabular}


Tabelle XVII.

Versuche bei einer Versuchstemperatur von $2^{\circ} \mathrm{C}$.

\begin{tabular}{c|c|c|c|c}
\hline \hline $\begin{array}{c}\text { Schüttelzeit } \\
\text { Minuten }\end{array}$ & $\begin{array}{c}\text { Koagulationszeit } \\
\text { Minuten }\end{array}$ & $(\mathrm{a}-\mathrm{x})$ & $\mathrm{k}_{\mathbf{1}}$ & $\mathrm{k}_{\frac{\mathbf{3}}{\mathbf{2}}}$ \\
\hline 0,0 & 9,52 & - & - & - \\
1,0 & 19,4 & 49,1 & 0,31 & 0,43 \\
2,0 & 36,15 & 26,3 & 0,29 & 0,47 \\
3,0 & 62,0 & 15,4 & 0,27 & 0,52 \\
4,0 & 88,35 & 10,8 & 0,24 & 0,51 \\
5,0 & 144,0 & 6,6 & 0,24 & 0,58 \\
6,0 & 197,5 & 4,8 & 0,22 & 0,59
\end{tabular}

Tabelle XVIII.

Versuche bei einer Versuchstemperatur von $3^{\circ} \mathrm{C}$.

\begin{tabular}{c|c|c|c|c}
\hline \hline $\begin{array}{c}\text { Schüttelzeit } \\
\text { Minuten }\end{array}$ & $\begin{array}{c}\text { Koagulationszeit } \\
\text { Minuten }\end{array}$ & $(\mathrm{a}-\mathrm{x})$ & $\mathrm{k}_{\mathbf{1}}$ & $\frac{\mathrm{k}_{\mathbf{3}}}{\mathbf{8}}$ \\
\hline 0 & 7,07 & - & - & - \\
0,75 & 14,95 & 47,3 & 0,43 & 0,61 \\
1,0 & 19,8 & 35,7 & 0,45 & 0,67 \\
1,5 & 31,75 & 22,3 & 0,44 & 0,74 \\
2,0 & 51,25 & 13,8 & 0,43 & 0,85 \\
2,5 & 97,5 & 7,3 & 0,45 & 1,09 \\
3,0 & 134,3 & 5,3 & 0,43 & 1,12
\end{tabular}

Es ist aus den angeführten Werten für $(\mathbf{a}-\mathbf{x})$ sowie von den Reaktionskonstanten ersichtlich, daß die *Schüttelinaktivierung " mit der steigenden Temperatur zunimmt, und zwar in der Weise, daß sie für etwa $20^{\circ}$ Temperatursteigerung verdoppelt wird.

Da die Reaktionskonstante, wie mehrmals hervorgehoben, wegen des unsicheren Reaktionsverlaufes nur approximativ berechnet werden darf, ist eine Berechnung der Temperaturkonstante, $\mu$, der Arrheniusschen Formel

auch nur annähernd.

$$
\frac{\mathrm{k}_{\mathrm{T}_{1}}}{\mathrm{k}_{\mathrm{T}_{9}}}=\mathrm{e}^{\frac{\mu}{2}\left(\frac{\mathrm{T}_{1}-\mathrm{T}_{2}}{\mathrm{~T}_{1} \cdot \mathrm{T}_{2}}\right)}
$$


Wir wählen zu dieser Berechnung die mittleren Werte der Konstante $\mathrm{k}_{\frac{3}{2}}$ und berechnen:

$$
\begin{aligned}
& \text { zwischen } 0 \text { und } 10^{\circ} \text { C. } \mu=6500
\end{aligned}
$$

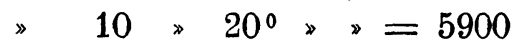

$$
\begin{aligned}
& \text { » } 20 \text { » } 30^{\circ} \text { » }=8800
\end{aligned}
$$

Wenn für das Temperaturintervall $20-30^{\circ} \mathrm{G}$, , wo die Reaktion sich vielleicht dem Verlauf der monomolekulären Reaktionsformel nähert, die Temperaturkonstante mit Hilfe der mittleren Werte von $k_{1}$ berechnet wird, ergibt sich $\mu=9100$.

Die Temperaturkonstante für die Schüttelinaktivierung läßt sich demnach zwischen 0 und $30^{\circ} \mathrm{C}$. im Mittel zu etwa 7000 berechnen. Zum Vergleiche erwähnen wir, daß für die Hitzedestruktion des Labs Madsen und Walbum Werte bis zu 90000 fanden, und für die Lichtdestruktion fanden wir ${ }^{1}$ ) früher etwa 1500 .

\section{Einfluß verschiedener Faktoren.}

Es war uns bei unseren ersten Versuchen aufgefallen, daß die Labpräparate des Handels (verschiedene norwegische Käselabextrakte und das Gladsche Labpulver), wenn sie nach hinreichender Verdünnung direkt zu den Schüttelversuchen verwendet wurden, sich nicht «inaktivieren, ließen. Da diese Präparate bekanntlich durch Extraktion mittels verdünnter Salzsäure bereitet sind und, um haltbar zu sein, mit reichlichen Mengen von Kochsalz und Borsäure versetzt sind, veranlaßte uns diese Beobachtung, den Einfluß verschiedener Elektrolyten, namentlich Säuren, zu untersuchen.

Diese Untersuchungen, ${ }^{2}$ ) die der Zeitschrift f. physikalische Chemie eingereicht worden sind, zeigten, daß ganz geringe Mengen von Säuren, z. B. $0,5-1,0 \mathrm{ccm} \mathrm{n/10-HCl}$ auf $100 \mathrm{ccm}$ Lablösung, hinreichend sind, um beim Schütteln in 5 Minuten jede «Schüttelinaktivierung » zu verhindern. Verschiedene organische Säuren, wie Oxalsäure und Milchsäure, üben eine

1) Signe und Sigval Schmidt-Nielsen, 1. c. S. 246.

?) Signe und Sigval Schmidt-Nielsen, cÜber den Einfluß der Säuren auf die Schüttelinaktivierung des Labs> (erscheint im Sommer 1909). 
442 Schmidt-Nielsen, Über «Schüttelinaktivierung > des Labs. I.

ebenso kräftige Wirkung wie die Schwefelsäure aus, und diese und andere organische Säuren wirken kräftiger hindernd als die Phosphorsäure. Aus unseren diesbezüglichen Versuchen geht hervor, daß die Säurewirkung nicht mit der Zahl der Wasserstoffionen proportional ist und somit nicht durch die Wirkung dieser erklärt wird. Untersuchungen über den Einfluß anderer Elektrolyten wie Nichtelektrolyten sind schon im Gange und werden demnächst veröffentlicht.

Zum Schluß möchten wir in dieser ersten Mitteilung ein eigentümliches Verhalten erwähnen, nämlich, daß die "Schüttelinaktivierung» unter gewissen Umständen einen reversiblen Prozeß darzustellen scheint. Hierauf, wie auf andere Versuche, die wir angestellt haben, um die ganze Erscheinung zu erklären, werden wir in einer zweiten Mitteilung eingehen. 